\section{AB001. Minimally invasive surgery for centrally located lung cancers}

\author{
Tangbing Chen, Jizhuang Luo, Chunyu Ji, \\ Feng Yao, Wentao Fang
}

Department of Thoracic Surgery, Shanghai Chest Hospital, Jiaotong University Medical School, Shanghai 200030, China

Correspondence to: Wentao Fang, MD. Department of Thoracic Surgery, Shanghai Chest Hospital, Jiaotong University Medical School, 241

Huaihai Road West, Shanghai 200030, China.

Email: vwtfang@hotmail.com.

Abstract: Lung cancers are currently the most frequently diagnosed malignancies and the leading cause of cancer death. Clinically they are divided into centrally located and peripheral ones according to their position on imaging study. With the advent of surgical techniques, early stage lung cancers, especially those peripherally located, are increasingly resected via minimally invasive approaches. These include both video-assisted thoracoscopic surgery (VATS) and robotic surgery. Its advantages over open thoracotomy include less pain, decreased postoperative complications, less impaired pulmonary function, and better tolerance of adjuvant therapies and improved quality of life after surgery. And similar oncologic outcomes in lymph node dissection and long-term survival have been witnessed in minimally invasive surgery (MIS) and in open surgery. Therefore, it is now strongly recommended for surgical management of lung cancers. For centrally located lung cancers, however, most of them are still resected via open surgery, especially those lesions requiring extensive resections such as sleeve lobectomy or pneumonectomy. Although the first cases of VATS pneumonectomy and VATS sleeve lobectomy were reported by Walker et al. in 1994 and by Santambrogio et al. in 2002, respectively, there are still major concerns on technical difficulties, safety, and oncological outcomes which hinder the acceptance of MIS in the treatment of centrally located lung cancers. There have been a few individual cases reported on successful sleeve lobectomy accomplished via MIS. It is only recently have small series studies comparing MIS versus open sleeve lobectomy appeared, all of them from China. In general, their results showed that MIS sleeve resections were feasible, although longer operation time was required comparing to open surgery. Conversion rates were unanimously quite low in these series. And the limited prolongation in operation time might be translated into better peri-operative results, including less intraoperative bleeding, decreased post-operative drainage, and shortened hospital stay. In addition, there are emerging data showing that long-term outcomes after MIS sleeve lobectomy may be comparable to open procedures, making it an appearing selection for patients with centrally located lung cancers. Similarly there have already been a number of successful cases reporting the feasibility of MIS for pneumonectomy in patients with central lung cancers. In the limited number of small case series comparing pneumonectomies via MIS or open surgery, operation time was again longer under MIS. And conversion rates were unsurprisingly higher than in MIS sleeve lobectomy, indicating that pneumonectomy under MIS was even more technically demanding. However, peri-operative patient benefit was also more obvious with MIS for pneumonectomy than for sleeve lobectomy. This was manifested in less intraoperative bleeding, less postoperative pain and fewer surgical complications. And the recently published National Cancer Database study found comparable peri-operative mortality rates and equivalent 5-year survival rates between MIS and open pneumonectomy for lung cancer patients. These at least indicate that pneumonectomy performed by minimally invasive approaches does not compromise peri-operative or long term outcomes. To prove the efficacy of MIS in either sleeve lobectomy or pneumonectomy for centrally located lung cancers, it is imperative to validate that oncological outcomes are at least non-inferior to standard open surgery. Besides, it is also necessary to show enough peri-operative benefits so as to make prolonged operation time worthwhile. For these purposes, we retrospectively reviewed our experience with MIS, including both VATS and robotic, sleeve lobectomies and pneumonectomies at the Shanghai Chest Hospital during the previous decade. First a propensity-score matched study was carried out comparing 79 cases of MIS versus 258 cases of open sleeve lobectomy (1:4 matching). Operation time was significantly longer in the MIS group (185.72 \pm 69.17 minutes) than in the open group $(148.68 \pm 40.51$ minutes, $\mathrm{P}<0.001)$. No significant difference was found in intra-operative bleeding, complete resection rate, number of lymph nodes dissected, or number of positive lymph nodes detected between the two groups. Postoperative drainage volume was significantly less in the MIS group $(1,376.95 \pm 865.91 \mathrm{~mL})$ than in the open group 
$(1,991.29 \pm 1,397.51 \mathrm{~mL}, \mathrm{P}<0.001)$. Although postoperative complication rates were similar between the two groups, there were $30(11.63 \%)$ patients in the open group with postoperative stay longer than 14 days, but only 4 (5.06\%) in the MIS group $(\mathrm{P}=0.09)$. Three-year overall survival rates (69.7\% vs. $77.2 \%, \mathrm{P}=0.27)$ and cumulative recurrence rates ( $35.2 \%$ vs. $28.8 \%, \mathrm{P}=0.20$ ) were both similar in the MIS group and in the open group. Upon multivariable analysis, pathological $\mathrm{T}$ stage and $\mathrm{N}$ stage were the independent risk factors for overall survival, and pathological $\mathrm{N}$ stage was the only independent risk factor for disease recurrence, while surgical approach was not related to oncological outcomes. Operation time, intra-operative bleeding, postoperative drainage, and postoperative complication rate were similar between VATS and robotic sleeve lobectomies. Conversion rate was only $6.2 \%$ in this series. And the converted cases had similar peri-operative outcomes comparing to open surgery cases. Another propensity-score matched study compared 29 patients receiving VATS pneumonectomy and 112 patients having open pneumonectomy (1:4 matching). Again MIS was associated with significantly prolonged operation time $(222 \pm 56$ vs. $170 \pm 44, \mathrm{P}<0.01)$, but R0 rates $(86.6 \%$ vs. $83.9 \%$, $\mathrm{P}=0.697)$ were comparable between the two groups, with higher number of $\mathrm{N} 2$ lymph node resection via MIS (20.5 \pm 9.2 vs. $17.0 \pm 7.3, \mathrm{P}=0.053)$. Shortened duration of drainage
(5.6 \pm 2.3 vs. $7.3 \pm 3.5, \mathrm{P}=0.015)$ was observed after MIS than after open surgery. But no difference was found in overall complication rates $(18.8 \%$ vs. $22.6 \%, \mathrm{P}=0.617), 30$-day mortalities $(2.7 \%$ vs. $3.4 \%, \mathrm{P}=0.871)$ or 90 -day mortalities ( $4.5 \%$ vs. $3.4 \%, \mathrm{P}=0.763)$, or length of hospital stay $(9.3 \pm 3.3$ vs. 13.6 $\pm 16.1, \mathrm{P}=0.140$ ) between the two groups. These results indicated that in well selected patients, both sleeve lobectomy and pneumonectomy could be safely achieved via MIS, including both VATS and robotic approaches. Prolonged operation time for MIS may be warranted, as it is associated with improved recovery manifested as decreased drainage and shortened hospital stay. Meanwhile, oncological principles are not compromised as there is no difference in complete tumor resection or extent of lymph node dissection. And similar survival and disease recurrence may be expected after MIS and open surgery for centrally located lung cancers.

Keywords: Minimally invasive surgery (MIS); thoracotomy; lung cancer; pneumonectomy; sleeve lobectomy

doi: 10.21037/shc.2019.AB001

Cite this abstract as: Chen T, Luo J, Ji C, Yao F, Fang W. Minimally invasive surgery for centrally located lung cancers. Shanghai Chest 2019;3:AB001. 